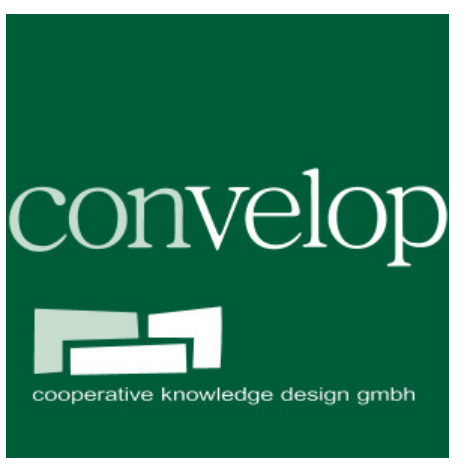

\title{
Evaluation des Pilotprogramms „Josef Ressel Zentren“"
}

Endbericht

$\begin{array}{ll}\text { Auftraggeber: } & \text { Bundesministerium für Wirtschaft, Familie und Jugend } \\ \text { Projektnummer: } & \text { 1000_JRZeval_1006 } \\ \text { AutorInnen: } & \text { Gabriele Gerhardter, Markus Gruber } \\ \text { Datum: } & 30.09 .2010\end{array}$




\section{Inhaltsverzeichnis}

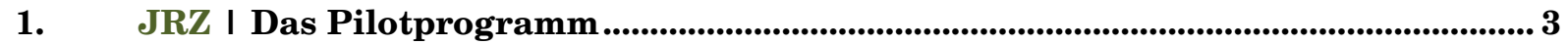

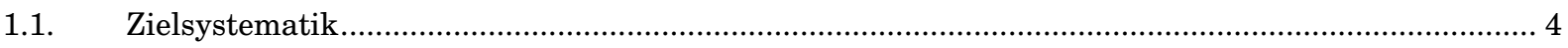

1.2. Prozesse und Organisation der Programmumsetzung (Förderungsabwicklung) .............................. 7

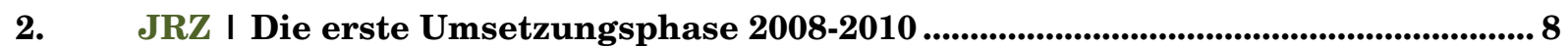

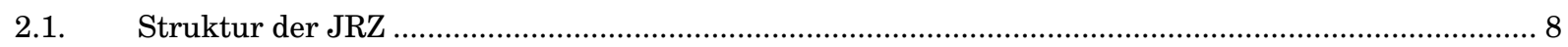

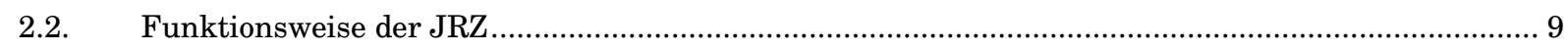

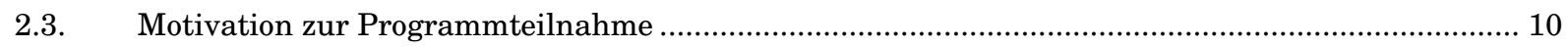

2.4. Anmerkungen zu einzelnen Gestaltungselementen ............................................................... 11

3. Exkurs I Fachhochschulen im „Strukturloch“ der Forschungspolitik.................. 14

4. JRZ I Schlussfolgerungen und Empfehlungen........................................................ 16

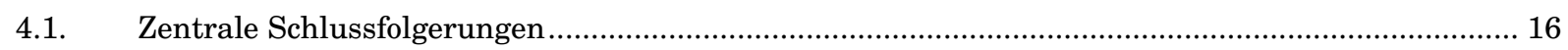

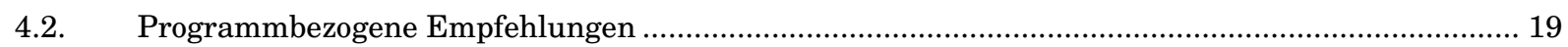

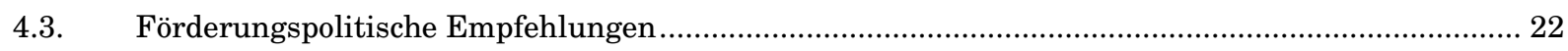

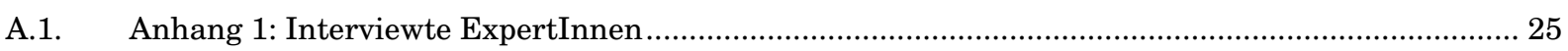

A.2. Anhang 2: Dokumenten-, Literatur- und Quellenverzeichnis ..................................................... 26 


\section{Einleitung}

Das Pilotprogramm Josef Ressel Zentren (JRZ) des Bundesministeriums für Wirtschaft, Jugend und Familie (BMWFJ) richtet sich an forschungserfahrene Fachhochschulen (FH's), die mit Unternehmen ein mehrjähriges Forschungsprogramm umsetzen. Derzeit gibt es drei Fachhochschulen, die ein JRZ eingerichtet haben:

- CFD Centre - Optimierung von gebäude-, energie- und umweltverfahrenstechnischen Prozessen mit Computational Fluid Dynamics [Fachhochschulstudiengänge Burgenland]

- Heureka! - Heuristische Optimierung [FH Oberösterreich]

- OptimUns - Optimierung unter Unsicherheit [FH Vorarlberg]

Das Ziel der Programmevaluierung, die von Juli bis September 2010 stattfand, war die Analyse der Konzeption und Prozesse des Pilotprogramms sowie die Unterstützung der strategischen Steuerung und Positionierung der JRZ für das BMWFJ anhand folgender Aspekte:

- Bewertung bisheriger Aktivitäten der JRZ und der Programmbetreuung durch die Österreichische Forschungsförderungsgesellschaft (FFG);

- Einschätzungen der Fachhochschulen und der beteiligten Unternehmen: Interviews mit den Projektleitern und den GeschäftsführerInnen der FH sowie mit Unternehmen, die im Konsortium in JRZ tätig sind;

- Einbettung in die forschungs-, technologie- und innovationspolitische Förderungslandschaft durch Analyse und Interviews mit FTI-politischen Akteuren.

Das Evaluierungsdesign basiert auf einer fundierten Auseinandersetzung mit dem Programmdokument und weiteren -unterlagen sowie Abbildung der Sichtweisen von unterschiedlichen Akteuren (Liste interviewter ExpertInnen im Anhang) auf das Gesamtprogramm. Dadurch sind eine ausgewogene Bewertung des Pilotprogramms und eine realistische Einschätzung in Bezug auf Adaptierungsbedarf und zukünftiger Positionierung gewährleistet. Für das BMWFJ sollen damit handlungsleitende Perspektiven und Gestaltungsinformationen über die Pilotphase hinaus ermittelt werden.

Die drei JRZ wurden im Zeitraum der Programmevaluierung nach ca. 1,5 bis 2jähriger Laufzeit einer, von der FFG organisierten, Zwischenevaluierung (2YE) unterzogen. Dies wurde insofern in der Programmevaluierung berücksichtigt, indem der Schwerpunkt verstärkt auf das Gesamtprogramm und nicht auf Ergebnisse der einzelnen Zentren gelegt wurde.

Die Programmevaluierung wirft einen systemischen Blick auf das Programm und ermittelt seine Funktion und bisherigen Effekte sowie Optimierungsbedarf auf Programmebene und förderungspolitische Einbettung. Analysiert werden die Positionierung des Programms, der Einfluss des JRZ-Programms auf Fachhochschulen und Unternehmen, sowie insgesamt der Fortschritt in den Bemühungen zur nachhaltigen Implementierung von Forschungskompetenz der Fachhochschulen mit der Wirtschaft. 


\section{JRZ | Das Pilotprogramm}

Die forschungsbezogene Zusammenarbeit zwischen Fachhochschulen und Unternehmen steht im Fokus des Forschungsförderungsprogramms „Josef Ressel Zentren“. In den letzten Jahren haben die Fachhochschulen u. a. durch das „FHplus Programm“ bzw. „COIN“ gezeigt, dass sie erfolgreiche Partnerschaften mit der Wirtschaft bilden können. Die JRZ sollen dies mit einem herausragenden Forschungsniveau in einer längerfristigen Kooperation mit Unternehmen und in breiter Einbindung von Lehre und Wissenschaft noch toppen. Das Pilotprogramm „Josef Ressel Zentren“ richtet sich demnach an FH's, die einen Forschung strukturell aufbauen wollen und hierbei sowohl das wissenschaftliche Potenzial als auch ein regionales Unternehmensumfeld aufweisen, das dazu in der Lage ist, an längerfristigen Forschungsvorhaben und Problemlösungen zu arbeiten. Eineinhalb bis zwei Jahre nach Start der ersten drei Zentren interessiert nun, inwieweit diese Zielsetzung zutrifft und die Programmausrichtung stimmt.

\section{Programmhistorie}

Die Idee von „Forschungslaboren“ an Fachhochschulen geht bereits auf das Jahr 2001 zurück, als die FH's noch relativ neue Einrichtungen im tertiären Bildungssektor in Österreich waren. Der Impuls kam jedoch nicht von Seiten der FH's, vielmehr wollten Industrieunternehmen Fachhochschulen in ihrer Region als längerfristige Forschungspartner einbinden. Dies stärkte zunächst die Assoziation eines „Christian Doppler Labors $^{1}$ an der $\mathrm{FH}^{\text {“ }}$ und damit auch die Verknüpfung zur Christian Doppler Gesellschaft (CDG) als Umsetzungseinrichtung. Langjährige interne Diskussionsprozesse in der CDG und dem, auch für die CDG zuständigen, Ministerium BMWFJ (damals BMWA) führten zu dem Ergebnis, dass für die „Forschungslabore“ der FH's das Modell der CDG nicht passend sei. Somit wurde die FFG mit der Entwicklung eines Programmes für Forschung an den Fachhochschulen in Kooperation mit der Wirtschaft beauftragt. Von der FFG wurde ebenfalls das Programm „FHplus“ bzw. als Nachfolgeprogramm „COIN Aufbauvorhaben“ betreut, das den Forschungsstrukturaufbau von Fachhochschulen unterstützt. Die „Forschungslabore an FH's“, die nun „Josef Ressel Zentren“ genannt wurden, sollten dabei die Forschungsaktivitäten der FH's intensivieren und den Unternehmen exzellente Forschungsangebote in der Region bieten. Zielgruppe sind somit „forschungserfahrene“ FH's, die bereits über entsprechende Forschungsinfrastruktur und -knowhow verfügen. 2008 erfolgte die erste Programmausschreibung im Wettbewerb, zwischen Oktober 2008 und Jänner 2009 starteten die ersten drei Josef Ressel Zentren im Pilotprogramm.

\section{Programmdesign}

Die Ausschreibung des Pilotprogramms erfolgte mit folgenden Eckpunkten: (tabellarischer Kurzüberblick nächste Seite)

${ }^{1}$ Christian Doppler Labor: siehe www.cdg.ac.at 


\begin{tabular}{l|l} 
Bereich & Bedingungen \\
\hline Einreichende & $\begin{array}{l}\text { Forschungserfahrene Fachhochschule } \\
\text { studiengangs- und erhalterübergreifende Einreichung möglich }\end{array}$ \\
\hline Partner & $\begin{array}{l}\text { Mind. 1 KMU, weitere Unternehmenspartner erwünscht } \\
\text { Assoziierte Partner aus dem Wissenschaftsbereich erwünscht }\end{array}$ \\
\hline Laufzeit & $\begin{array}{l}\text { Maximal 5 Jahre } \\
\text { Nach 2 Jahren erfolgt eine Zwischenevaluierung }\end{array}$ \\
\hline Förderungshöhe & $\begin{array}{l}\text { max. 350.000 Euro Bundesförderung pro Zentrum auf 2 Jahre } \\
\text { die F\&E-Infrastrukturnutzung darf nicht mehr als 20\% betragen }\end{array}$ \\
\hline Förderungs- & $\begin{array}{l}\text { max. 40\% Bundesförderung } \\
\text { mind. 10\% Fachhochschule } \\
\text { mind. 50\% Unternehmenspartner }\end{array}$ \\
\hline Inhaltlich & $\begin{array}{l}\text { Thema des JRZ entspricht einem Entwicklungsschwerpunkt der FH } \\
\text { Unforwiegende Durchführung von Forschenden an der FH } \\
\text { Forschungsprogramm in gemeinsamer Abstimmung mit FH und } \\
\text { Unternehmenspartnern } \\
\text { Hoher wissenschaftlicher Anspruch (state of the art) sowie konkreter } \\
\text { Nutzen für die Unternehmenspartner } \\
\text { Förderung des Ausbaus des wissenschaftlichen Nachwuchses inkl. } \\
\text { Rückkoppelung in die Lehre, Masterarbeiten, Dissertationen }\end{array}$ \\
\hline $\begin{array}{l}\text { Programmverantwortlich: BMWFJ } \\
\text { Programmbetreuung: FFG }\end{array}$ \\
\hline Programm-
\end{tabular}

\subsection{Zielsystematik}

Die Förderung von Forschungskooperationen zwischen Fachhochschulen und Partnern aus Wirtschaft und Wissenschaft im Rahmen der Josef Ressel Zentren soll den forschungsintensiven Wissens- und Technologietransfer in der Region vorantreiben. Im Wesentlichen ergeben sich aus dem Programmdokument drei Programmziele:

- Erstes Programmziel ist die Etablierung einer stabilen, längerfristigen Kooperationsbeziehung der FH mit der Wirtschaft in der Region.

- Das zweite Programmziel besteht in der Stärkung der Forschungsfähigkeit bei Unternehmen, die Zugang zu fundierter wissenschaftlicher Expertise erhalten und somit ihre Produkte und Prozesse optimieren und innovieren können.

- Das dritte Programmziel beinhaltet die Entwicklung von Forschungskompetenz an der FH. Die Erkenntnisse der JRZ müssen in das Ausbildungsangebot der FH einfließen - dies betrifft sowohl die Lehre als auch die F\&E-Arbeiten. Grundlagenbezogene Forschungsfragen sollen speziell durch eine Kooperation mit Universitäten bearbeitet werden. Insgesamt soll F\&E mit einem hohen Anspruch an Exzellenz und damit der Ausbau von Forschungsgruppen an FH's unterstützt werden. 
Das Programmdokument definiert nicht explizit, jedoch implizit drei Zielgruppen der JRZ:

1. Längerfristige Kooperationsbeziehungen zur Wirtschaft - direkte Zielgruppe: Unternehmen;

2. Forschungskompetenz an den FH's nutzen und vertiefen - direkte Zielgruppe: Fachhochschulen;

3. Längerfristige Kooperationsbeziehungen zu Universitäten - indirekte Zielgruppe: Universitäten (eine Zusammenarbeit mit wissenschaftlichen Partnern wird gewünscht, jedoch nicht monetär gefördert).

Das Programmdokument der Josef Ressel Zentren orientiert sich im Aufbau am „COIN“Programmdokument und trifft in manchen Formulierungen und Zielvorgaben zu wenig die spezifischen Ziele, Funktionen und Leistungsbereichen der JRZ - hier sollten in Zukunft noch eine Schärfung der Ziele und klare Erwartungsanforderungen erfolgen, nicht wie bisher vor allem über die Indikatoren, sondern als Zieldefinitionen. Dies betrifft $u$. a. zwei Formulierungen, die zum besseren Verständnis der weiteren Evaluationsergebnisse bereits hier einer Klärung unterzogen werden:

1. Stärkung der Forschungsfähigkeit der Unternehmen: Die an den JRZ beteiligten Unternehmen sind bereits „forschungsfähig“, sie finden Forschung für das Unternehmen wichtig und haben z.T. bereits Erfahrungen mit Forschungsförderungsprogrammen. Durch die Beteiligung am JRZ verändert sich jedoch die Forschungsintensität. Die Unternehmen nehmen an längerfristigen Forschungsprozessen (mehr als F\&E-Projekte) teil, die andere Zeithorizonte und Logiken haben und oftmals breitere Lösungen beinhalten.

2. Die Bezeichnung "grundlagenorientiert“ passt genauso wenig wie der Begriff „Forschungslabor" auf die Forschungsinhalte der JRZ. JRZ sind vielmehr im Bereich einer „wissenschaftsintensiven, anwendungsorientierten Forschung“ tätig. Dies bedeutet, dass sie den Forschungsprozess akademisch organisieren (Forschungsgruppen), sich auf neueste wissenschaftliche Grundlagen und Erkenntnisse (state of the art) beziehen und diese für die Anwendung in Unternehmen aufgreifen, adaptieren und konkret einsetzbar machen. Dazu benötigen sie auf wissenschaftlicher Seite die Einbindung in die Scientific Community und die Kenntnis über neueste Forschungsergebnisse, auf Unternehmensseite den Zugang zu Daten und Informationen. Somit geht es für die JRZ auch nicht um den Eintritt ins Konkurrenzfeld der „wissenschaftlichen Exzellenz“, sondern um ein Höchstmaß der wissenschaftsintensiven Forschung an Fachhochschulen in Kooperation mit Unternehmen - dies ist derzeit ein sehr hoher Anspruch für die FH-Landschaft in Österreich.

Ein „logic chart“ des Pilotprogramms „Josef Ressel Zentren“ zeigt, in welchem Zusammenhang Ziele, Instrumente, Output- und Outcome gesehen werden. Die „strichlierten“ Elemente verweisen darauf, dass diese implizit im Programmdokument enthalten sind, jedoch zu wenig explizit hervortreten. 
Abbildung: „logic chart“ des Pilotprogramms „Josef Ressel Zentren“, convelop 2010

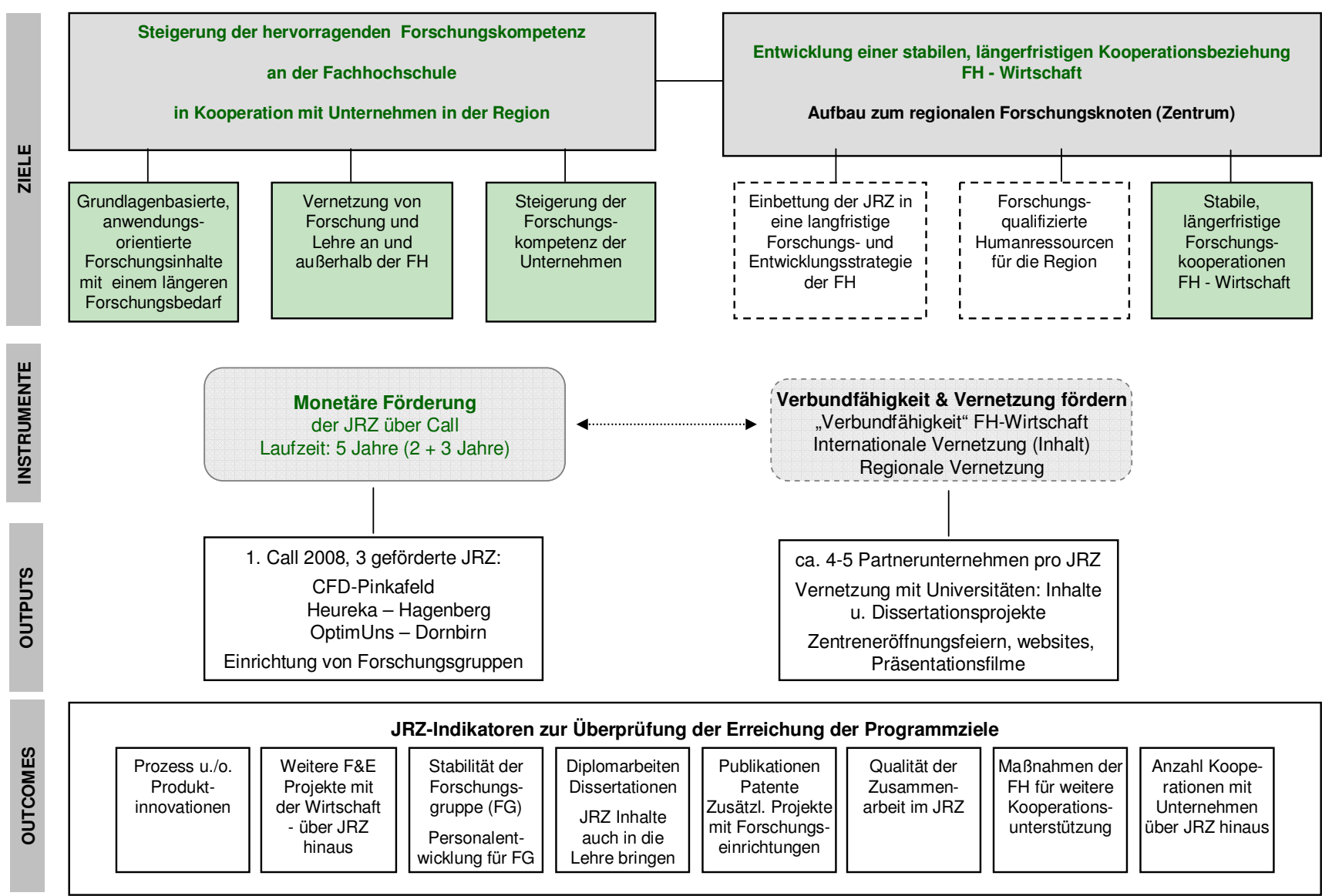

Anmerkung:

Anmerkung:

"Ziele: schwarze Schrift 
Die explizite Integration des Zieles „Aufbau eines regionalen Forschungsknotens“ mit Begleitaktivitäten sowie die konsequente Einbindung in die langfristige Forschungsund Entwicklungsstrategie der FH (und ihrer Erhalter) würden die „Zentrumsqualität“ vermehrt betonen. Dazu werden auch - über die Forschungsaktivitäten hinausgehende - Maßnahmen zur Stärkung der der „Verbundfähigkeit“2 der FH mit der Wirtschaft benötigt (siehe dazu Kapitel Empfehlungen).

\subsection{Prozesse und Organisation der Programmumsetzung (Förderungsabwicklung)}

Das Pilotprogramm "Josef Ressel Zentren" wird von der Österreichischen Forschungsförderungsgesellschaft betreut. Diese war bei der Entwicklung des Programmdesigns dem BMWFJ behilflich und ist im Folgenden für die Information, Programmeinreichung und -abwicklung sowie Betreuung der Zentren zuständig.

Für die Programmabwicklung steht ein Budget von 10\% der Programmkosten zur Verfügung, wobei der Start des Programms davon bereits viele Ressourcen benötigte. Unabhängig vom Programmvolumen entstehen set-up Kosten (Erstellung des Informationsmaterials, elektronischer Einreichung, Juryauswahl und -durchführung), die im selben $\mathrm{Ma} ß$ aufwendig sind als bei einem monetär größeren Programm, wodurch bei einem kleinen Programm die Abwicklungskosten zu Beginn relativ höher sind. ${ }^{3}$

\section{Folgende Prozesse lassen sich in der Programmabwicklung darstellen:}

1. Nach Bekanntmachung der Ausschreibung hatten die FörderungswerberInnen vom 18.02.2008 bis 11.04.2008 (8 Wochen) Zeit, einen Antrag einzureichen.

2. Es wurden 6 Anträge eingereicht. Die Anträge wurden in Bezug auf formale Kriterien durch die FFG geprüft.

3. Die Anträge wurden durch je zwei externe wissenschaftliche FachgutachterInnen aus dem deutschsprachigen Raum innerhalb von drei Wochen inhaltlich begutachtet (jeweils ein/e GutachterIn aus dem FH-Bereich, ein/e GutachterIn aus dem universitären Bereich pro Antrag).

4. Eine Jury gab auf Grundlage der Anträge und der Fachgutachten eine Förderungsempfehlung für das BMWFJ ab. Es wurden drei Anträge zur Förderung empfohlen.

5. Die Entscheidung über die Förderung der JRZ wurde durch das BMWFJ gefällt.

6. Im Anschluss daran erfolgte die Absage an nicht zu fördernde EinreicherInnen sowie die Vertragserstellung mit den geförderten EinreicherInnen durch die FFG. Die Vetragserstellung hat sich aufgrund von Ausfällen von Unternehmenspartnern (Höhepunkt der Wirtschaftskrise Herbst 2008) und Suche nach neuen Partnern zeitlich bei zwei Antragstellern verzögert.

7. Die laufende Betreuung inkl. Einforderung des Berichtswesens sowie Auszahlung der Förderung erfolgt durch die FFG, ebenso die Organisation der Zentrenevaluierung (2YE) im Zeitraum Früh- bis Spätsommer 2010.

\footnotetext{
${ }^{2}$ Verbundfähigkeit ist mehr als Kooperation: Kooperation ist die projektbezogene Zusammenarbeit, Verbundfähigkeit ist die potenzielle Zusammenarbeitsmöglichkeit, d.h. den Forschungsbedarf und das -angebot untereinander zu kennen und bei gegebenen Anlass projektbezogen zu aktivieren.

3 Generell zu überlegen wäre, ob für gewisse „Fixbestandteile“ der Programmabwicklung Fixkosten sinnvoller sind (z.B. Erstellung eines Leitfadens, Einrichtung der elektronischen Einreichung etc.), lediglich für die laufende Betreuung der FörderungsnehmerInnen sowie dem Controlling einen Prozentwert anzusetzen ist.
} 


\section{JRZ | Die erste Umsetzungsphase 2008-2010}

\subsection{Struktur der JRZ}

Im ersten Call zum JRZ-Programm wurden 6 Anträge eingereicht, 3 JRZ wurden in einem Juryverfahren ausgewählt und starteten Mitte 2008 mit der Programmumsetzung. Zur Fördersumme von 2.015.000 Euro über fünf Jahre ${ }^{4}$ in der ersten Ausschreibung kommen 795.500 Euro an geldwerten Leistungen der Fachhochschulen und 1.299.000 Euro an Barleistungen der Unternehmenspartner plus ebensoviele in-kind Leistungen hinzu. Der weitaus größte Finanzierungsbeitrag der Partner aus der Wirtschaft stammt von Großunternehmen. Auch wenn in den JRZ KMU mitwirken, sind deren finanzielle Beiträge in Summe eher gering.

Die maximale Bundesförderung der JRZ beträgt 40\%, die jedoch von einem JRZ nicht voll beantragt wird. Der Finanzierungsanteil der FH liegt bei zwei FH's bei exakt 10\% (Mindestvoraussetzung), eine FH übernimmt einen größeren Finanzierungsanteil am JRZ.

Als Unternehmenskooperation ist mindestens $1 \mathrm{KMU}$ gefordert - tatsächlich haben die JRZ 4 bis 6 Unternehmenspartner, wobei die Anzahl der KMU's in der Minderheit (1 bis $2 \mathrm{KMU}$ ) ist. Die Unternehmenspartner hatten teilweise vor Antragseinreichung bereits Kooperationsbeziehungen mit der FH, ca. 1/3 konnte für die JRZ Kooperation neu gewonnen werden.

Der Bezug zur Region wird in zwei JRZ direkt zum Bundesland gesehen, das JRZ im Burgenland hat hier - aufgrund der Betriebsstruktur des Burgenlandes und der räumlichen Lage - ein weiteres Einzugsgebiet über drei Bundesländer (Bgld, NÖ, Stmk).

Mit den JRZ werden Forschungsgruppen an den Fachhochschulen mit ca. 4 bis 5 Vollzeitäquivalenten (VZÄ) aufgebaut. In der Regel verteilen sich diese auf 1-2 Vollanstellungen und bis zu zehn Teilzeitmitarbeitenden (inklusive studentischer Mitarbeit).

Die Finanzierung des JRZ wird überwiegend - entsprechend der Vorgaben im Programmdokument - zum Aufbau der Personalressourcen eingesetzt (in einem JRZ zu 100\%).

Forschungsschwerpunkt aller drei geförderter Zentren ist der Bereich „Optimierung“. Dies wird „Zufall“ sein, zeigt jedoch, dass wissenschaftsgeleitet „Methoden und Verfahren“ erarbeitet werden können, die in Zukunft für andere Forschungs- und Entwicklungsprojekte ebenso zur Verfügung stehen. Aus Unternehmenssicht wurde dazu berichtet, dass derzeit die Optimierung in Unternehmen mit am Markt verfügbaren Angeboten an ihre Grenzen stößt, sodass es neuer, grundlegender Lösungen bedarf und sich dazu ein JRZ mit seiner längerfristigen und wissenschaftsintensiven Forschungsangebot besonders eigne.

\footnotetext{
${ }^{4}$ Die JRZ verpflichten sich mit Antrag auf einen Umsetzungszeitraum mit entsprechender Finanzierung über fünf Jahre- daher wird hier die Finanzierung insgesamt dargestellt, nicht nur für die ersten beiden Jahre.
} 


\subsection{Funktionsweise der JRZ}

Interviews mit den JRZ Leitern, den GeschäftsführerInnen bzw. einem Forschungsleiter der FH's und Partnerunternehmen zeichnen nach nicht ganz zweijähriger Laufzeit ein Bild der Funktionsweise der JRZ, das aus fünf Kernelementen der Forschungskooperation zwischen FH und Wirtschaft besteht.

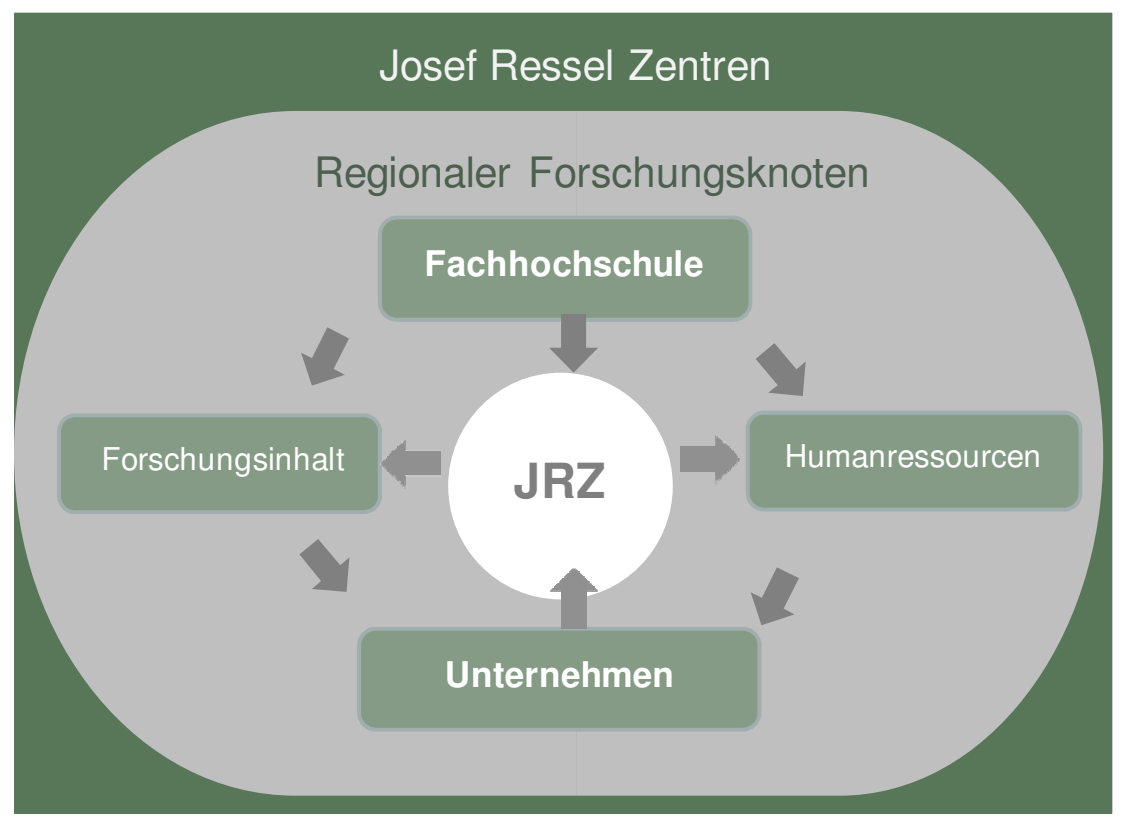

Abbildung: 5 Kernelemente der JRZ, convelop 2010

1. Fachhochschule: Die Fachhochschule muss in der Lage sein, hochwertige angewandte Forschung zu betreiben, d.h. entsprechende Infrastruktur und geeignetes Forschungspersonal aufweisen sowie Lehr- und Forschungsschwerpunkte im Themenbereich des JRZ haben. Entscheidend ist, wie die Lehre mit der Forschung verknüpft wird und diese gemeinsam zum Aufbau der strategischen Ausrichtung der FH dienen.

-> „forschungserfahrene $\mathrm{FH}^{\text {“ }}$ bedeutet: Eine $\mathrm{FH}$, die strategische Forschungsschwerpunkte langfristig etablieren und sich damit auch ein Profil geben will, somit entsprechende Ressourcen zur Verfügung stellt.

2. Unternehmen: JRZ benötigen eine regionale Unternehmensstruktur, die in der Lage ist, sich auf einen längeren Forschungsprozess einzulassen und dafür finanzielle Ressourcen zur Verfügung zu stellen. Häufig sind dies F\&E-betreibende Unternehmen, die mit ihren inkrementellen Entwicklungen anstehen und erkennen, dass sie nun wissenschaftsintensives Wissen benötigen. Entscheidend ist, dass die Unternehmen untereinander nicht in Konkurrenzsituation stehen, jedoch den gleichen Kern an Forschungsinteresse aufweisen.

3. Forschungsinhalte mit hohem wissenschaftlichen Niveau: Der Forschungsschwerpunkt muss sich am "state of the art" der Forschung orientieren. Es soll dadurch ein Qualitätsniveau ermöglicht werden, das neue Lösungen im wissenschaftlichen Feld und Unternehmensbereich ermöglicht. Wissenschaftlichkeit orientiert sich dabei in der globalen Vernetzung - hier müssen die FH's und die 
Unternehmen anschlussfähig gemacht werden. Geeignet sind jene Inhalte, die breit übertragbar sind, $d$. h. Lösungen für mehrere Unternehmenspartner im JRZ bieten und auch in Zukunft Wissenstransfer - insbesondere für KMU - in der Region ermöglichen.

4. Humanressourcen für die Forschung: Das JRZ muss Entwicklungsoptionen für die Forschenden in unterschiedlichen Bereichen bieten: den JRZ LeiterInnen weitere Andockmöglichkeit in der wissenschaftlichen Community, den qualifizierten ProjektmitarbeiterInnen die Möglichkeit für Publikationen und Anbindung an die Unternehmensforschung und für die Studierenden die Möglichkeit zur Mitarbeit, Masterarbeit, Dissertationen sowie erste Publikationen inkl. Eintrittsmöglichkeit in eine industrielle oder wissenschaftliche Karriere. MitarbeiterInnen der Partnerunternehmen lernen, am Forschungsprozess beteiligt zu sein bzw. wissenschaftsintensives Wissen für Unternehmensinnovationen „abzuholen“.

5. Regionaler Forschungsknoten: Der erste Schritt für den Aufbau eines regionalen Forschungsknotens besteht darin, die oben dargestellten vier Kernelemente effektiv und effizient miteinander zu verknüpften, um hier synergetisch zu wirken. Der zweite Schritt beinhaltet die Herstellung einer „Verbundfähigkeit“ der FH - als aktiver Wissenspartner für Unternehmen in der Region. Dies ist nicht alleine über Forschung zu erzeugen, diese ist jedoch ein wichtiger Faktor darin. Die Erzeugung von Sichtbarkeit der Wissens- und Transferleistungen unterstützt die Entwicklung in Richtung regionaler Forschungsknoten. Interface für einen regionalen Forschungsknoten ist nicht das JRZ, sondern die Fachhochschule - das JRZ stimuliert jedoch und treibt das Interface an.

Die JRZ in der derzeitigen Pilotphase funktionieren mit ihren vielfältigen Anforderungen vor allem durch das starke Engagement der JRZ Leiter, die über Hindernisse (wie z. B. Dissertationsmöglichkeiten für Mitarbeitende im JRZ) durch persönliche Kontakte hinweg helfen oder aufgrund ihrer bisherigen ausgezeichneten wissenschaftlichen Karriere das Vertrauen der Unternehmen gewinnen, hier wertvolle Lösungen zu erhalten. Mittelfristig soll es jedoch gelingen, hier strukturelle Effekte zu erzielen, indem Forschungskapazität in der Region aufgebaut und diese strategisch und institutionell eingebunden wird.

\subsection{Motivation zur Programmteilnahme}

Das Pilotprogramm JRZ wurde quasi in einer Linie und Ergänzung zum „FHplus Programm" weiterentwickelt und stellt die nächste ambitionierte Forschungsstufe für die FH's dar. Die Zielgruppe FH war demnach sehr gut bekannt, deren Bedarfe, Möglichkeiten und Interessen ebenso. Dennoch interessiert es speziell bei einem 1. Call, welche Motivationen zur Teilnahme die Zielgruppen haben.

Die Teilnahme der Fachhochschulen erfährt vor allem durch drei Faktoren ihre Motivation:

1. Langfristige, wissenschaftsintensive Forschung: Die FH's möchten einen Forschungsschwerpunkt aufbauen, in dem über einen längeren Zeitraum 
Forschungsinhalte verfolgt werden. Sie möchten dabei von einer „perforierten“ (über einzelne Projekte) zu einer „kontinuierlichen“ Forschung gelangen, die auch über entsprechendes Personal verfügt.

2. Verbesserung des Angebots für die Studierenden: Die Verknüpfung des Forschungsangebotes mit der Lehre sowie die Möglichkeiten der studentischen Mitarbeit an Projekten, Diplomarbeiten und Dissertationen bietet den Studierenden ein interessantes Angebot, dass über „verschulte“ Strukturen der FH hinausweist.

3. Anreicherung durch die Unternehmenspraxis: Die Kooperation mit den Unternehmen wird als wichtiger Input für Forschung und Lehre erachtet und soll zu weiteren Kooperationen führen.

Partnerunternehmen an den Josef Ressel Zentren motivieren vor allem zwei Faktoren zur Teilnahme am Programm:

1. Wissenschaftsintensive Forschung: Die an JRZ beteiligten Unternehmen haben einen Bedarf an wissenschaftsintensiver Forschung. Ein Unternehmen formulierte dies folgendermaßen: „Wir sind an der Grenze angekommen: mit am Markt verfügbaren Lösungen kommen wir in der Optimierung nicht mehr weiter - wir müssen dazu grundsätzlicher forschen, damit hier noch Verbesserungen möglich sind“.

2. Regionales Angebot der Forschungskooperation: Viele Partnerunternehmen der JRZ haben bereits Forschungserfahrung - bei Programmen der FFG und auf EUEbene. Sie alle heben insbesondere den regionalen Aspekt der JRZ hervor - die Verfügbarkeit vor Ort, die direkte Ansprache, das gemeinsame Verständnis und auch, dass die gemeinsame Forschungsleistung letztendlich der regionalen Wertschöpfung zu Gute kommt. 5

\subsection{Anmerkungen zu einzelnen Gestaltungselementen}

\section{Langfristigkeit des Programms als Hürde und Chance}

Die Möglichkeit, über fünf Jahre an einem wissenschaftsintensiven Forschungsprogramm zu arbeiten, wurde von den FH's positiv aufgefasst, für die Unternehmen stellt dies durchaus eine Motivationshürde dar, da Vorstände und Geschäftsführungen überzeugt werden müssen, über den gesamten Zeitraum ein Budget für den Forschungsbereich zur Verfügung zu stellen. Speziell für KMU's ist dies eine kritische Hürde, die Teilnahmen auch verhindert hat (siehe dazu auch Kapitel Empfehlungen).

Die Sinnhaftigkeit der Langfristigkeit des Forschungsvorhabens ist mit drei Argumenten zu unterstreichen:

1. Lediglich mit langfristigen Programmen kann wissenschaftsintensive anwendungsorientierte Forschungskompetenz an der FH aufgebaut werden. Damit ist es möglich, qualifiziertes Forschungspersonal zu erhalten, Ergebnisse in die Lehre einzubringen und das gewonnene Know-how auf längere Sicht auch in den Technologietransfer mit KMU fließen zu lassen.

\footnotetext{
${ }^{5}$ Dabei ist hier darauf hinzuweisen, dass die drei Zentren der Pilotphase ihren Standort jeweils in Regionen/Bundesländern ohne technische Universitäten haben, wodurch die regionale Nähe des Forschungspartners FH wohl nochmals an Bedeutung gewonnen hat.
} 
2. Die Unternehmen steigen in tatsächliche Forschungsprozesse (und nicht nur inkrementeller Entwicklung) ein, d. h. sie lernen, dass Forschung länger dauert, es eventuell Ideen gibt, von denen man sich verabschieden muss bzw. neue Lösungswege gangbar sind.

3. Nur mit langfristigen Programmen können entsprechende wissenschaftliche Vertiefung erfolgen, Dissertationsvorhaben und Publikationen getätigt werden, womit auch besserer wissenschaftlicher Außenauftritt und die Forcierung einer Forschungskarriere möglich wird.

Die Hürde der Langfristigkeit besteht somit vor allem in der Phase der Antragserstellung, in der die Unternehmenspartner von der Dauer der Kooperation überzeugt werden müssen. Während der Zusammenarbeit im JRZ bedeutet dies jedoch auch, einzelne Entwicklungsschritte und Ergebnisse für die Unternehmen immer wieder „sichtbar“ zu machen. In den Interviews unterstrichen die JRZ Partnerunternehmen und die JRZ Leiter, dass fünf Jahre einen guten Forschungsrahmen bieten, um hier flexibel reagieren zu können und zu wichtigen Ergebnissen zu kommen.

\section{Was ist das „Mehr“ am Zentrum im Vergleich zu einem Projekt?}

Die JRZ sind als „Zentren“ ausgeschrieben, nicht als Forschungsvorhaben. Dies begründet sich einerseits aus der fünfjährigen Förderungsdauer, andererseits aus der Funktion, langfristig zur Etablierung eines regionalen Forschungsknotens beizutragen. Ein Zentrum ermöglicht einen besseren Außenauftritt, klare Kommunikation und breites Zur-Verfügung-Stellen des Leistungsangebotes sowie Planungssicherheit. Der Begriff „Zentrum“ ist für die JRZ wichtig,

- da gewährleistet wird, einen Forschungsbetrieb an der FH aufzubauen, der über einzelne, verstreute Projekte hinausgeht und dafür auch entsprechendes Personal zu gewinnen bzw. aufzubauen;

- da das Ziel der wissenschaftsintensiven Forschung nicht ein Produkt ist, sondern eine „Methodik“, die multiplizierbar ist und damit auch in Zukunft für Wissenstransferleistungen an Unternehmen, z. T. auch in anderen „Märkten“ als den ursprünglichen Entwicklungsfeldern zur Verfügung steht;

- er schafft eine längerfristige wissenschaftliche Basis und Forschungsorientierung für die FH, die Einfluss auf die Lehre ausübt sowie Möglichkeiten für Masterarbeiten und Dissertationen bietet;

- er ermöglicht der FH, wissensintensive anwendungsorientierte Forschung zu betreiben - und nicht ausschließlich „auftragsgetriebene“ Forschung - dies gekoppelt mit Unterstützung der strategischen Ausrichtung und Profilbildung der FH;

- er verlangt einen sichtbaren Außenauftritt und ein offensives Angebot der FH als Forschungspartner für die Wirtschaft.

Die Folgewirkungen des Zentrumsbegriffs sind bei der Programmerstellung nicht vollständig bedacht bzw. ist ihnen zu wenig Aufmerksamkeit geschenkt worden. Ein Zentrum stellt eine längerfristige Infrastruktur dar, die entsprechende Stabilität verlangt, die über fünf Jahre hinausgeht - diese ist bei Antragsbeginn nicht sichergestellt bzw. wird der Frage der „Nachhaltigkeit“ der JRZ noch zu wenig Aufmerksamkeit geschenkt. 


\section{- Erfolgreiche JRZ durch „creaming“ in der FH-Landschaft}

Ein JRZ kann dann gut funktionieren bzw. eine FH bei Antragsstellung für ein JRZ erfolgreich sein, wenn Folgendes gegeben ist:

- Eine klare Forschungsstrategie der FH inkl. Aufbau von Schwerpunkten, Personalbesetzung, Einbindung von Studierenden und Infrastruktur.

- Eine Etablierung der FH als Entwicklungspartner für Unternehmen in der Region, d. h. es gibt bereits Projekte bzw. längerfristige Kooperationen und eine gute $\mathrm{Zu}$ sammenarbeit mit regionalen Leitbetrieben.

- Eine gute Vernetzung der JRZ LeiterInnen in der (internationalen) Scientific Community, Arbeitskenntnisse in Form von „Forschungsgruppen“ und die Möglichkeit, hier wissenschaftliche Anschlussfähigkeit der FH herstellen zu können, wobei bereits Prozesse zur Überleitung von „personalen“ zu „stukturellen“ Kooperationen eingeleitet worden sind.

- Die FH verfügt über ein Forschungs- bzw. Rahmenbudget, damit Vorleistungen insbesondere im Bereich der Infrastruktur und Begleitaktivitäten zur Profilbildung des JRZ und in Richtung regionaler Forschungsknoten getätigt werden können.

Die Anforderungen sind vielfältig und hoch, nicht alle FH's in Österreich haben entsprechende Voraussetzungen. Es werden daher nur bereits in der Forschung etablierte Fachhochschulen für ein JRZ in Frage kommen. Forschungsaktive FH's sind entsprechend interessiert, ein JRZ einzurichten, die somit Signalwirkung erhalten. Damit unterstützt das JRZ-Programm auch - im positiven Sinne - eine Profilbildung und Ausdifferenzierung der FH-Landschaft in Österreich.

\section{- JRZ - Forschung organisiert sich anders als in Förderungsschienen}

JRZ geraten mit ihrer wissenschaftsintensiven anwendungsorientierten Forschung in einen „diffusen“ Grabenkampf der institutionalisierten Forschungsförderungsschienen in Österreich. Vereinfacht folgen die beiden Schienen der linearen Verknüpfung:

- Universitäten - Grundlagenforschung - Industrie (Großunternehmen)

- Fachhochschulen - anwendungsorientierte Forschung - KMU

Diese beiden „Schienen“ finden sich in Wissenschafts- und Forschungspolitik sowie der Förderungspraxis wieder. Es wird immer wieder versucht, hier „Brücken“ zu schlagen, die zumeist einem „Tanz auf dem Eis“ der Zuständigkeiten gleichen (siehe Exkurs). Die Forschungsrealität hat das „Schienendenken“ bereits längst überholt: die Partnerunternehmen der JRZ sind überwiegend Großunternehmen, ihnen war es „egal“ ob ihnen eine Universität oder eine FH die Forschungsleistung anbietet. Nationale wie internationale Universitäten arbeiten mit den JRZ ohne Vorbehalte wissenschaftlich zusammen: Forschung organisiert sich manchmal anders, als die (Förderungs-)Politik es will. 


\section{Exkurs | Fachhochschulen im „Strukturloch“ der Forschungspolitik}

Fachhochschulen in Österreich haben einen Forschungsauftrag, dieser ergibt sich mehr indirekt als direkt aus dem Fachhochschulstudiengesetz (FHStG):

- $\quad \S 3$ Abs 1 und Abs 2: In dieser Bestimmung wird das Studienprofil der Fachhochschulen in Form von leitenden Grundsätzen normiert. Es wird darauf hingewiesen, dass es sich um Studien auf Hochschulniveau handelt, die dem Grundsatz der Einheit von Wissenschaft und Lehre folgen. Der Begriff „wissenschaftlich fundierte Berufsausbildung" wird dazu verwendet. Abs 2 führt weiter aus, dass die Vielfalt der wissenschaftlichen Lehrmeinungen und der wissenschaftlichen Methoden zu beachten sind.

- $\quad \S 5 \mathrm{a}$ hält fest, dass an Fachhochschulen Lehr- und Forschungspersonal tätig ist.

- $\quad \$ 12$ Abs 2 Z 4 verpflichtet die Fachhochschulen folglich, die zur Erreichung der Ziele und zur Sicherung der Grundsätze erforderlichen anwendungsbezogenen Forschungs- und Entwicklungsarbeiten durch Mitglieder des Lehr- und Forschungspersonals durchzuführen.

- $\quad \$ 16$ Abs 6 legt fest, dass die Erhalter der Fachhochschulen dafür zu sorgen haben, dass das Lehr- und Forschungspersonal an anwendungsbezogenen Forschungs- und Entwicklungsarbeiten teilnimmt. Dies kann in der eigenen Einrichtung oder durch Kooperation mit anderen Forschungs- und Entwicklungseinrichtungen geschehen.

Zuständig für die Fachhochschulen ist das Bundesministerium für Wissenschaft und Forschung (BMWF). Anders als an den Universitäten ist die Finanzierung der Fachhochschulen durch den Bund nicht im Gesetz geregelt (vgl. für Universitäten im § 12 UG 2002), sondern in den Fachhochschul Entwicklungs- und Finanzierungsplänen, die seit 1994 jeweils für einen Zeitraum von vier Jahren abgeschlossen und vom Ministerrat verabschiedet werden. Die Finanzierung laut Plan erfolgt auf Basis der Studienplatzbewirtschaftung nach dem Normkostenmodell, Forschungsfinanzierung ist dabei nicht vorgesehen.

Wer ist jedoch für die Finanzierung der Forschung an Fachhochschulen zuständig? Hier beginnt der Kreislauf der wechselseitigen Zuweisung der Zuständigkeiten:

- BMWF (Bundesministerium für Wissenschaft und Forschung): erklärt sich zuständig für die gesetzlichen Regelungen, Akkreditierung und Studienplatzförderung der FH's, jedoch nicht für die Forschungsfinanzierung, da diese ,angewandte" Forschung ist und somit in den Bereich der FH-Erhalter bzw. in die Kompetenzen der Bundesländer sowie des BMWFJ und BMVIT fällt.

- BMVIT (Bundesministerium für Verkehr, Innovation und Technologie): ist verantwortlich für die Förderung anwendungsorientierter Forschung. FH's können an BMVIT (ko-)finanzierten Programmen als Forschungsakteur teilnehmen, das Ministerium sieht sich jedoch nicht als Förderstelle für Fachhochschulen zuständig. 
Das BMVIT hat zur Stimulierung der FH's als aktive Forschungsakteure die „Impulsaktion Kooperation Fachhochschulen -Wirtschaft“" (Ausschreibung 1997, 1999, 2000) und anschließend das Programm „FHplus“ (2002, 2004) aufgelegt und sieht jetzt an der Beteiligung der $\mathrm{FH}^{\prime}$ 's an zahlreichen anderen Programmen (insbesondere COIN und Bridge), dass die damit intendierten Ziele erreicht wurden.

- BMWFJ (Bundesministerium für Wirtschaft, Familie und Jugend): fördert Forschung in Kooperation mit der Wirtschaft und hat dazu einige Programme aufgelegt wie z. B. CD-Labors, Laura Bassi Centres, Research Studios und fördert im Rahmen von COIN-Aufbau die Fachhochschulen (gemeinsam mit BMVIT) sowie seit 2008 die Josef Ressel Zentren. Forschungspolitische Strategie ist die Stimulierung der Forschungsleistungen für Unternehmen und Standort.

- FH-Erhalter respektive Länder: Für Forschung ist in Österreich der Bund zuständig. An zahlreichen FH's sind die Länder die Erhalter, die z. T. die Forschung an der FH mit einer Art „Basisfinanzierung“ (unterschiedlicher Art und Höhe einige mit direkten „Forschungsbudgets“, andere mit „Rahmenbudgets“, aus denen auch Forschungsaktivitäten finanziert werden können) ausstatten, wie z. B. in Oberösterreich, Vorarlberg, Steiermark, neuerdings Salzburg und ein wenig Burgenland, ebenso Kärnten. Die Länder verweisen jedoch auf die generelle Forschungszuständigkeit des Bundes und erachten hier Unterstützungsbedarf.

Fachhochschulen gibt es in Österreich seit 1994, wobei in den letzten Jahren starke Veränderungen und Ausdifferenzierung in der FH-Landschaft erfolgten. Derzeit studieren rund $36.000^{6}$ Studierende in Österreich (ca. 13\% aller Studierenden) an einer FH, jährlich gibt es ca. 14.000 StudienanfängerInnen. Die Anzahl der Studierenden wächst, die Bedeutung der FH's für die außeruniversitäre Forschung ebenso. Dass FH's in der Forschung aktiv sind, zeigen die zahlreichen Beteiligungen an Forschungsförderungsprogrammen der FFG. Der - theoretisch anmutende - Streit, wie anwendungs- bzw. grundlagenorientiert diese Forschung ist, wird durch die Praxis längst eingeholt. Es gibt nicht entweder „grundlagenorientiert“ oder „anwendungsorientiert“, sondern viele Aspekte dazwischen, in denen Forschung an den FH’s - Großteils in Kooperation mit Unternehmen - passiert.

Die JRZ stehen im Prozess der Beobachtung der Veränderung und Ausformulierung von Verantwortung der Hochschullandschaft in Österreich. Es sieht so aus, dass Forschung an den FH's hier noch eine langfristige Herausforderung in einem fortlaufenden Prozess sein wird. Für Fachhochschulen braucht es auf Seiten der Forschungsförderung insgesamt noch ein Konzept - derzeit befindet sie sich in einem „Strukturloch“.

${ }^{6}$ Angaben laut uni:data (aktuellste Angabe: Wintersemester 2009) 


\section{JRZ | Schlussfolgerungen und Empfehlungen}

Das JRZ-Programm war zum Startzeitpunkt förderungspolitisch isoliert, in keiner Programmschiene eingepasst und zudem vom „Strukturloch der Zuständigkeiten“ um Forschung an der FH betroffen, somit zunächst mit einer zweijährigen Pilotphase versehen. Eine Programmevaluierung nach zwei Jahren soll nun Klarheit über die Fortführung bzw. Optimierung des Programms geben.

\subsection{Zentrale Schlussfolgerungen}

\section{- Prinzipielle Passfähigkeit zwischen Zielen und Umsetzung ist gegeben}

Das Förderungsprogramm wirkt im einem Bereich, der in den letzten zwölf Jahren aufgebaut wurde und nun spezifische Schwerpunktsetzungen benötigt: die forschungsbezogene Kooperation von Fachhochschulen mit der Wirtschaft. Hier besteht noch Entwicklungsbedarf hin zu wissenschaftsintensiven Forschungsinhalten und effizienter, nachhaltiger Verbundfähigkeit sowohl auf Seiten der FH's als auch der Unternehmen. Es zeigt sich nach der bisher noch nicht ganz zweijährigen Umsetzungsphase der JRZ sowohl auf Zentrenebene (2YE) als auch auf Programmebene - dass die zentralen Zielsetzungen des Programms passend sind und in der Gesamtlaufzeit erreicht werden können.

\section{- Eine Mobilisierungswirkung für die Zielgruppen ist durch die elitäre Ausrichtung erreicht}

Das JRZ-Programm dient der Verstärkung der „Wissenschaftsintensivierung“ in der angewandten Forschung, bietet damit den regionalen Unternehmen neuartige Wissensangebote und stärkt die Profilbildung an den FH's. Nur ein kleines, exquisites Programm kann gewährleisten, dass der hohe Anspruch die FH's in ihren Forschungsleistungen anspornt und diese auch mit der Lehre und den regionalen Bedarfen verknüpft. Wichtig dabei ist eine strenge Evaluierung der Anträge bezüglich der Forschungsinhalte, der verfügbaren Infrastruktur, des Forschungspersonals und der Unternehmenskooperationen mit der Frage: Bringen diese ausgezeichnete, wissenschaftsintensive und anwendungsorientierte Forschung hervor? Sonst besteht die Gefahr, dass die wissenschaftliche Basis schnell erodiert. Eine „Auswahl der Besten“ ist durch das Antragsverfahren (Call) gegeben (ein paar Anmerkungen zu mehr „Treffsicherheit“ gibt es im Kapitel Empfehlungen). Das Programm motiviert FH's mit einem Forschungsschwerpunkt und entsprechender Infrastruktur, wissenschaftsintensive anwendungsorientierte Forschung in Kooperation mit Unternehmen zu tätigen und mobilisiert die FHLandschaft hin zu einem sehr hohen Niveau der angewandten Forschung. Die Fixierung im Programm auf KMU als Unternehmenspartner muss dabei - wie die Praxis zeigt aufgegeben werden, da gerade KMU wenig Kapazität für fünfjährige Forschungsprozesse haben. Daher eignen sich regionale Leitbetriebe besser zur Kooperation. Die Unternehmen werden dahingehend motiviert, längerfristige Forschungskooperationen einzugehen und hier wissenschaftsintensiver zu arbeiten. Die regionale Einbindung ist für die Unternehmen dabei wesentlich (auch diejenigen Unternehmen, die bereits mehr Forschungserfahrung haben, betonen den regionalen, persönlichen Charakter des JRZ), „Ansprechpartner vor Ort“" zu haben, ist dabei entscheidend. 


\section{- Sehr gute Relation zwischen Mitteleinsatz und bisherigen Ergebnissen bzw. zu erwartenden Erfolgen}

Die zeitliche und finanzielle Dimensionierung der JRZ wurde richtig gewählt, um eine Betonung der wissenschaftlichen anwendungsorientierten Forschung zu erreichen und damit auch Signale für die regionale Wissensbasis zu setzen. Eine Bundesförderung von max. $40 \%$ (max. $350.000 €$ für 2 Jahre) erreicht hier vielfältige Impulse und Effekte auf FH-, Unternehmens- und regionaler Ebene, die weitere Forschungsaktivitäten stimulieren und stützen. Von den InterviewpartnerInnen wurde betont, dass die fünfjährige Laufzeit den Fachhochschulen eine gute Basis für fundierte Forschungsarbeiten mit einer mittelfristigen Planungssicherheit bietet und dies ein gerade noch zu überschaubarer Forschungszeitraum für Unternehmen darstellt. Neben den direkten Forschungsleistungen entstehen „indirekte“ positive Effekte:

- Den JRZ kommt eine Signalfunktion in Richtung „Fachhochschule als Forschungsknoten" in der Region zu. Dies hat bereits im einem Fall eine Betriebsansiedlung in der Region unterstützt, da hier sowohl Forschungsstrukturen als auch entsprechend qualifizierte Humanressourcen vor Ort verfügbar sind.

- Das JRZ-Programm leistet einen positiven Anreiz zur Profilbildung innerhalb der Forschungslandschaft Österreichs durch seine wissenschaftliche Basis, v. a. durch die Vernetzung mit wissenschaftlichen Partnern, durch Publikationstätigkeiten und Dissertationsmöglichkeiten.

- Positive Effekte auf KMU entstehen nicht ausschließlich durch die unmittelbare Beteiligung am JRZ, sondern auch indirekt durch die daran anschließenden Entwicklungsprojekte sowie durch die Rückkoppelung in die Lehre und damit ein „Upgrading“ bzw. mehr „Realitätsnähe“ der Ausbildung, somit zukünftiger Arbeitskräfte.

- Programmdokument (Antrag) braucht noch mehr Präzision der Ziele und Funktion der JRZ bei prinzipiell mehr Offenheit der Zielerreichung (siehe auch Kapitel: Zielsystematik sowie Empfehlungen zum Programmdesign)

Das Programmdokument sollte insgesamt in den Zielen und Funktionen des JRZ sowie in Bezug auf die Nachhaltigkeit des Programms geschärft werden. Insbesondere die Bedeutung der Humanressourcenentwicklung sowie die regionale Funktion der JRZ (siehe Kapitel „Funktion der JRZ“) müssten noch entsprechend hervorgehoben werden.

Es zeigt sich durch die Evaluierung, dass die Kooperationsbeziehungen zu den Universitäten lediglich mittelbare Ziele sind, die durch das Programmdesign, den Anspruchsgrad des Forschungsinhaltes und die universitäre Einbindung bzw. Herkunft der JRZ Leiter entstehen. Ein funktionsstärkeres, direktes Ziel der JRZ wäre hingegen „Aufbau eines regionalen Forschungsknotens“ in der Region - dies in zwei Dimensionen:

1) Forschungsqualifizierte Humanressourcen für die Region

- Als gezielte Nachwuchsförderung für Forschung an der FH, indem Studierenden die Mitarbeit an konkreten Forschungsvorhaben ermöglicht wird und aktuelle Inhalte in die Lehre einfließen - somit den Unternehmen in der Region ein forschungsqualifiziertes Personal in Zukunft zur Verfügung steht.

- Bezüglich Personal in den kooperierenden Unternehmen, die Forschungsleistung mitentwickeln bzw. begleiten, hier mehr Know-how erwerben und auch 
in Zukunft mehr forschungsintensives Wissen und ebenso Personal nachfragen werden.

- Im Sinn von „brain gain“ für die Region: Die JRZ sind ein attraktives Angebot für (universitäre) ForscherInnen, eine Forschungsgruppe an der FH aufzubauen und hier langfristig tätig zu sein. So werden attraktive Forschungsarbeitsplätze auch außerhalb von forschungsintensiven Ballungssräumen geschaffen, die die Bildungs-, Forschungs- und Unternehmenslandschaft bereichern.

2) Einbindung der JRZ in eine langfristige Forschungs- und Entwicklungsstrategie der $\mathrm{FH}$

- JRZ als Forschungssignal der FH: Ein JRZ unterstützt die Strategiebildung der FH und schärft ihr Profil, indem sie ihre Forschungsstärken präsentiert. Das bringt FH's in einen Prozess der Selektion: nicht alle Forschungsbereiche können auf hohem Niveau weiterentwicklelt werden, nur diejenigen, die über ein ausgeprägtes Forschungsknow-how verfügen, auf entsprechende Forschungsbedarfe der Unternehmen treffen und die Entwicklungsstrategie der FH unterstützen.

- Ein JRZ benötigt weitergehende Unternehmenskooperationen der FH: Eine Weiterführung der Forschungsinhalte der JRZ nach Ablauf der Förderungszeitraumes bedarf umfassende Unternehmens- und Forschungskooperationen, die durch die JRZ entwickelt und fortgeführt werden. Von der Verbundfähigkeit der FH soll jedoch auch das JRZ profitieren, indem dessen Ausbau und Wachstum unterstützt wird.

Die FH's sollten im Antrag aufgefordert werden, ihre Leistungen im Bereich der Ziele und Funktionen des JRZ klar darzustellen - bei prinzipieller Offenheit, wie sie dies erreichen möchten und in welchem Umfang (z. B. zeigen die Indikatoren zur Zielerreichung, dass sie bisher zu „tief“ gegriffen haben - alle Zentren haben im Bereich Publikationen, Dissertationen etc. die Zielsetzungen bereits übertroffen). Es erweist sich jedoch auch, dass ein zusätzliches Projekt mit der Wirtschaft zum Zeitpunkt der 2YE in einem JRZ aufgrund seiner Wissenschaftsorientierung noch zu früh ist. Hier sollten die JRZ im Bereich „Nachhaltiger Zentrumsaufbau und Anschlussoptionen“ darstellen, wie sie dies zu einem späteren Zeitpunkt erreichen wollen. In einem wettbewerblichen Verfahren werden dabei die besten Gesamtlösungen ausgewählt.

\section{- Anschlussoptionen für und Nachhaltigkeit der JRZ als ungeklärte Frage}

So gut die Ergebnisse der 2Y-Evaluierungen der einzelnen JRZ sind, so positiv sich erste Ergebnisse der JRZ auf Forschung, Lehre und regionale Wirtschaft einschätzen lassen: offen bleibt die Frage: Was passiert nach den fünf Jahren geförderter Laufzeit? In einem Programm, das wissenschaftsintensive, angewandte Forschungsqualität in einem Zentrum fördert, gilt der Frage der Nachhaltigkeit besondere Aufmerksamkeit.

Es kann nicht sein, dass

- die JRZ plötzlich beendet und „aufgelöst“ werden - dann wären es langfristige Projekte und keine Zentren, die intendierten weiteren, insbesondere regionalen Effekte würden verpuffen; 
- die JRZ im Anschluss auf „einfache“ Projekte (wie z.B. in COIN, Bridge) zurückgreifen. Dazu wären sie vor der fünfjährigen Förderung bereits fähig gewesen, würden diese somit obsolet machen.

Wenn die Intention verfolgt wird, dass FH's mit JRZ wissenschaftsintensiv arbeiten und hier eine spezifische Forschungsleistung für Unternehmen in der Region bereitsstellen, dann braucht es auch ein längerfristiges Bekenntnis zu diesen Zentren. Es ist daher zu überlegen, wie dieses nachhaltig unterstützt werden kann. U. a. auch, ob im Falle weiterer Programmausschreibungen auch bestehende Zentren antragsberechtigt (mit entsprechend strengen Evaluierungskriterien) sein können, wenn sie Wachstums- und Erweiterungsperspektiven verfolgen.

Denkbar ist ebenfalls, anspruchsvolle, wissenschaftsintensivere Projekte zu generieren - wie z. B. K-Projekte oder Beteiligung am Forschungsrahmenprogramm der EU. Dies löst noch nicht die Frage der Bedeutung als „Zentrum“ mit der Verknüpfung zur Lehre an der FH und der Verbundwirkung mit den regionalen Unternehmen.

In jedem Fall soll der Frage der Anschlussoption für die JRZ in den Jahren 3 bis 4 der Förderung besondere Aufmerksamkeit gewidmet werden und hier gemeinsam mit der FH (Erhalter) ein Konzept entwickelt werden.

\subsection{Programmbezogene Empfehlungen}

Die Ziele und Ausrichtungen des JRZ-Programms sind grundsätzlich passend und werden von der Zielgruppe verstanden. Hier folgen auf Basis der Interviews mit den Zentren und den Unternehmen sowie der Analyse der Dokumente Empfehlungen für Adaptierungen in einzelnen Bereichen.

- Das Antrags- und Begutachtungsverfahrens ist zeiteffizient, transparent und professionell - Optimierungsbedarf in wenigen Punkten.

- Das Ausschreibungsverfahren entspricht den FFG Standards bei wettbewerblichen Einreichungen und wurde entsprechend sorgfältig durchgeführt.

- Die Kriterien für die Beurteilung der Anträge sind transparent, die Auswahl der zu fördernden Anträge ist nachvollziehbar und sie wird entsprechend durch Fachgutachten und Juryempfehlungen begründet.

- Der Ausschreibungszeitraum war für die Einreichenden zu kurz gewählt, da insbesondere Kooperationen mit Unternehmen, die über fünf Jahre gehen, längere Entscheidungsprozesse innerhalb der Unternehmen benötigen. Es können damit auch kaum neue Forschungskooperationen aufgebaut, sondern weitgehend bestehende formalisiert werden.

- Die FachgutachterInnen wurden sorgfältig ausgewählt. Die Einschränkung auf den deutschsprachigen Raum ergibt sich aufgrund der Einreichsprache „Deutsch“.

- Die Jurymitglieder wurden in Zusammenarbeit mit dem BMWFJ festgelegt. Um eine klare Trennung zwischen Jury zur „Förderungsempfehlung“ und Ministerium zur „Förderungsentscheidung“ zu wahren, wird empfohlen, in Zukunft Jurymitglieder aus dem programmverantwortlichen Ministerium lediglich eine beratende 
Funktion ohne Stimmrecht zu geben; Jurymitglieder mit Stimmrecht sollten ausschließlich externe FachexpertInnen sein.

- Die Dauer des Verfahrens von Einreichschluss bis zur Förderungsempfehlung durch die Jury war angemessen, die Förderungsentscheidung durch das Ministerium ließ zu lange auf sich warten.

- Die inhaltlichen und administrativen Anforderungen an den Antrag sind angemessen und werden auch von den geförderten JRZ als passend gesehen.

- Die Anforderungen an das Berichtswesen und die Abrechnung der Fördermittel werden prinzipiell als angemessen erachtet. Es sind jedoch für die JRZ zwei Ausführungsverträge vorgesehen: für die Jahre 1-2 und die Jahre 3-5. Dies beinhaltet einen abrechnungstechnischen Mehraufwand, da hier jeweils „Endabrechnungen“ $\mathrm{zu}$ stellen sind, Kostenverschiebungen daher faktisch unmöglich gemacht werden und ein aufwendiges „finales Abrechnungsprozedere“ in der FFG eingeleitet wird. Dieses Prozedere schränkt die notwendige Flexibilität eines mehrjährigen Forschungsprojektes ein und verursacht zusätzliche Kosten auf Seiten der Abwicklung, die durch 5jährige Vertragslaufzeiten verhindert werden können.

Sehr positiv erwähnt wird von den JRZ-Leitern die ausgesprochen gute Zusammenarbeit mit der FFG als Förderungsstelle, die Information und Unterstützung in allen Fragen bietet. Insgesamt wird die Programmerstellung und -abwicklung - auch von Jurymitgliedern und weiteren Akteuren - als „sehr gut, professionell und sehr zielführend im Prozess“" (Interviewzitat mit einem FTI-Akteur) gesehen.

\section{- Präzisierung der Ziele und Funktionsbereiche des JRZ bei gleichzeitiger Offenheit in der Leistungserbringung.}

Im Antrag sollen die Leistungen der FH zu allen fünf Funktionen des JRZ (siehe Kapitel Funktionen des JRZ) ausführlich dargestellt werden, wobei es hier Offenheit in der Leistungserbringung (wie viele Unternehmen zusätzlich eingeworben, wie viele Publikationen entstehen, wie viele wissenschaftliche Partner einbezogen werden etc.) geben soll. Bisherige Indikatoren der Leistungsüberprüfung sind „zu tief“ gegriffen, hier sind die FH's für sich anspruchsvoller. Wichtig ist die Gesamtdarstellung, wie hier im Zusammenwirken der einzelnen Komponenten wissenschaftsintensive angewandte Forschung in Kooperation mit den Unternehmen in der Region erfolgreich und nachhaltig betrieben werden kann.

\section{Der Zentrumscharakter des JRZ soll stärker betont werden.}

JRZ sollen sich klar von einem Projekt oder einem „Labor“ unterscheiden und dies soll auch über Förderungsmöglichkeiten (z. B. über Begleitstrukturen, Öffentlichkeitsarbeit etc.) abgebildet werden. Hier bedarf es entsprechender „Begriffsbereinigungen“ auf Programmebene (Josef Ressel Zentren - Forschungslabore für die FH - dies passt nicht zusammen und weckt lediglich unglückliche Konnotationen zu CD-Laboren, wobei JRZ andere Funktionen haben). Die Gestaltung als „Zentrum“ soll auch bei Antragstellung beschrieben werden, insbesondere die Einbeziehung in die strategische Schwerpunktbildung der FH und in Bezug auf die Nachhaltigkeit nach Beendigung der Förderung. 
- Ein JRZ benötigt einen „multi-firm“ Kooperationsansatz (mind. 3 Unternehmen) mit gleichzeitig mehr Flexibilität in der Einbindung von KMU’s.

Der Anspruch der Beteiligung von mind. $1 \mathrm{KMU}$ im Programmdokument ist in zweifacher Weise nicht sinnvoll:

- Dies würde bedeuten, dass es auch ein „single-firm“ JRZ geben kann, welches weiteren Funktionen und Zielsetzungen des JRZ, insbesondere im regionalen Bereich, widersprechen würde. JRZ entwickeln i.d.R. eine unternehmensübergreifende „Methodik“, die es ermöglicht, in den Folgejahren auch am JRZ nicht beteiligten Unternehmen (insbesondere KMU's) qualitätsvolle F\&E-Dienstleistungen auf TopNiveau zu diesem Thema zur Verfügung zu stellen, ohne dass diese die langjährige Forschungsarbeiten mitfinanzieren mussten. Ein „single-firm“ Ansatz kann nicht die Breite der Forschungsinhalte garantieren, sodass die Übertragbarkeit für andere Unternehmen bzw. Branchen kaum gegeben ist.

- Es ist schwierig, KMU's für 5jährige Forschungsvorhaben zu gewinnen, insbesondere Kleinunternehmen haben hier nicht die Perspektive und das Budget. Sinnvoller wäre es, auf die Zielgruppe der regionalen Leitunternehmen (ohne eigener Forschungsabteilung) zu zielen und gleichzeitig darzustellen, wie die Forschungsergebnisse in Hinkunft auch für KMU nutzbar werden (z.B. durch Wissenstransfermaßnahmen etc.). Die Beteiligung von KMU's am JRZ sollte als Anspruch bleiben (ohne Vorgabe der Anzahl), jedoch flexibler gehandhabt werden. So sollten diese nicht notwendigerweise die gesamten fünf Jahre teilnehmen müssen, sondern flexibel einsteigen können (z.B. lediglich 2 Jahre teilnehmen).

\section{- Eine Öffnung für alle thematischen Forschungsrichtungen der FH's wird empfohlen.}

Die Einschränkung auf „wissenschaftlich-technische“ Forschungsinhalte ist heute nicht mehr nachvollziehbar. Da die Bedingungen für alle Einreichenden dieselben sind, wird hier der hohe wissenschaftlich Anspruch und die Forderung nach Finanzierung der Unternehmen für alle gefordert - dies gilt dann auch im Wirtschafts-, Sozial-, Medizin-, Pflegebereich etc. und soll diese nicht ausschließen. Es geht um eine Öffnung hin zu „sozialen Innovationen“. Dies ist insbesondere vor dem Hintergrund einer zunehmenden Technologisierung des Dienstleistungssektors von Bedeutung, wobei vielfach Schnittstellen zu technologischen Fragen abgedeckt werden können.

\section{- Studiengangsübergreifende Einreichung - ja, erhalterübergreifende Ein- reichung - nein.}

Eine erhalterübergreifende Einreichung würde sowohl die Profilbildung einer FH als auch die regionalen Effekte der JRZ unterminieren. Studiengangsübergreifende Einreichungen sind zu empfehlen, da diese sowohl den Forschungsschwerpunkt an der FH breiter unterstützen als auch vielfache Ergebnisse für die regionale Unternehmenslandschaft bieten können.

\section{- Maximal zwei Anträge pro Fachhochschulerhalter zur Einreichung.}

Die Festlegung auf maximal zwei Anträge pro Fachhochschulerhalter soll aufrecht bleiben. Die Differenzierungsprozesse in der FH-Landschaft sind soweit fortgeschritten, dass die „bekannten Förderprofis“ unter den FH's bei diesem kleinen Programm wahr- 
scheinlich alle Mitteln ausschöpfen würden. Damit wäre die faktische Anreizwirkung des JRZ-Programms auf die Forschungsintensität aller FH's in Österreich nicht mehr gegeben. Zudem ist es aufgrund der vielfältigen Funkionen eines JRZ für den FHErhalter ausreichend anspruchsvoll, zwei JRZ parallel aufzubauen.

\section{- Das JRZ-Programm könnte mit einem Ansatz der „dynamischen Zentren- gestaltung“ an Passgenauigkeit für FH, Unternehmen und Region ge- winnen.}

Derzeit müssen die JRZ bis zur Zwischenevaluierung (nach 1,5 bis 2 Jahren) ein zusätzliches Projekt eingeworben haben. Dieser Zeitraum wird für eine wissenschaftsintensive Forschungskooperation als zu früh erachtet, da der Schwerpunkt in dieser Phase auf der wissenschaftlich-methodischer Entwicklung liegt und noch zu wenig „sichtbare“ Ergebnisse bietet. Eine Einforderung zusätzlicher Projekte und Unternehmenspartner soll ab dem dritten Jahr passieren, ab diesem Zeitraum wäre auch die Beteiligung für KMU's einfacher. Ein Ansatz der „dynamischen Unternehmenskooperation“ würde auch beinhalten, dass die JRZ frei sind, ob sie weitere Kooperationspartner integrieren bzw. zusätzliche Projekte tätigen. Dies hätte selbstverständlich auch Auswirkungen auf die Planung, die diesbezüglich „Ressourcenreserven“ benötigt. Der Ansatz einer dynamischen Zentrengestaltung beinhaltet ebenso, dass im vierten Jahr der JRZ ein Strategiekonzept zur nachhaltigen Fortführung der JRZ über den Förderungszeitraum hinaus erarbeitet werden soll, dass an den bis zu diesem Zeitpunkt entwickelten Ergebnissen und Unternehmenskooperationen ansetzt und entsprechend weiter entwickelt wird.

\subsection{Förderungspolitische Empfehlungen}

Forschung braucht Wettbewerb und muss sich dem Wettbewerb stellen. Daher sollte es FH's mit ausgezeichneter Forschung geben, die ein Programm haben, dass sie zu wissenschaftsintensiver, angewandter Forschung bringt. Für die FH's ist das JRZProgramm dahingehend ausreichend aufwendig und auch herausfordernd.

Forschung an FH's lebt derzeit von Einzelprojekten, die mehr willkürlich sind (wo können wir ein Projekt einreichen?) und keine Planungssicherheit bezüglich Aufbau spezifischen Wissens und damit Verknüpfung mit einem kontinuierlichen Lehrangebot bieten. Mit den JRZ wird hier ein mittelfristiges Programm aufgelegt, das FH's anregt, strategische Forschungsschwerpunkte in Kooperation mit den Unternehmen aufzubauen. Derzeit noch mit großem personellen Engagement, in der Hoffnung, dass dies längerfristig auf struktureller Ebene wirkt.

Schwierig erscheinen momentan die Anschlussoptionen für die JRZ nach fünf Jahren Laufzeit. Dies insbesondere durch das beschriebene „Strukturloch“ der Forschungsförderung an FH's, das jedoch nicht zu Lasten der FH's, der Forschenden, Studierenden und auch der Unternehmen der Region gehen soll, sondern einen Klärungsauftrag auf FTI-politischer Ebene (Ministerien und auch Länder als Erhalter) beinhaltet. 
Die Programmevaluierung empfiehlt die Fortführung des Programms „Josef Ressel Zentren“ durch das BMWFJ als eigenständiges Programm.

Die Empfehlung basiert auf der Einschätzung der bisherigen Ergebnisse der JRZ, Erhebungen und Analysen im Rahmen der Programmevaluation und daraus gewonnenen Schlussfolgerungen, inkl. Hinweisen zur Adaptierung im Programmdesign.

\section{- Empfohlen wird die Fortführung des Programms}

- Dies betrifft die derzeitigen drei geförderten JRZ, die die erste Zwischenevaluierung mit sehr guten Bewertungen abgeschlossen haben und hier ihre Forschungstätigkeit die nächsten drei Jahre erfolgreich zu Ende bringen sollen. Bisherige Ergebnisse lassen auf weitere nachhaltige Effekte in der Kooperation mit der Wirtschaft in der Region schließen.

- Dies betrifft ebenfalls die weitere Durchführung von „Calls“. Aufgrund des hohen wissenschaftlichen Anspruchs des Programms sowie den Anforderungen an Infrastrukturbereitstellung, Vernetzung mit der Lehre und Einbindung von Unternehmenspartnern in Bezug auf den momentanen Stand der Forschungsstrukturen an FH's in Österreich, schätzen interviewte ExpertInnen sowie das Evaluierungsteam, dass es nicht mehr als 10 bis $12 \mathrm{JRZ}$ insgesamt in den nächsten Jahren geben kann, wenn dieses anspruchsvolle Niveau gehalten werden soll.

- $\mathrm{Zu}$ berücksichtigen wäre dabei auch die Option der Neueinreichung bestehender Zentren mit erweitertem Fokus. Einerseits geht es um die Nachhaltigkeit der geschaffenen Kapazitäten, die ohne einen entsprechenden wissenschaftsintensiven Ansatz wieder rasch in eher kurzfristig orientierte Entwicklungsaktivitäten übergehen. Andererseits kann so ein dynamisches Wachstum von Zentren und damit eine tatsächliche und anreizinduzierte Profilbildung in der österreichischen FH-Landschaft unterstützt werden.

- Empfohlen wird die Fortführung des Programms Josef Ressel Zenren durch das BMWFJ

- Das BMWFJ fördert die Wettbewerbsfähigkeit österreichischer Unternehmen, die wesentlich von Aktivitäten im Bereich von Forschung und Innovation abhängt. Dazu müssen Rahmenbedingungen zur Stärkung der Forschung geschaffen werden. Durch die Förderung der JRZ unterstützt das BMWFJ die Forschungsfähigkeit und -leistung der Unternehmen, insbesondere durch das hohe Niveau der angebotenen Forschungsleistungen, die Bereitstellung und Mitentwicklung der Humanressourcen für die Forschung als auch der regionalen Verfügbarkeit von Wissen und Forschung. Die Vernetzung der Unternehmen und die Verbundfähigkeit FH-Wirtschaft wird dabei gestärkt.

- Das JRZ übernimmt auch eine „pragmatisch“ wichtige Rolle zur Förderung von Forschung an FH's, insoweit es keine strukturelle Lösung der Forschungsfinanzierung an FH's (,forschungspolitisches Strukturloch“) gibt. Das JRZProgramm ist damit auch ein wichtiger, positiver Anreiz zur Profilbildung in der FH-Landschaft und zur Vernetzung der Forschung mit der Lehre. Das BMWFJ übernimmt hier z. T. auch eine „Zwischenfunktion“ in der Förderung, die wichtig ist, solange strategiegeleitete Forschung an der FH weiterhin mit strukturellen Unklarheiten zu kämpfen hat. 


\section{- Empfohlen wird die Fortführung der Josef Ressel Zentren durch das BMWFJ als eigenständiges Programm}

Forschungspolitisch steht außer Streit, dass Fachhochschulen in Österreich systematisch und kontinuierlich anwendungsorientierte Forschung betreiben, die den Bedarfen der regionalen Unternehmen entspricht. Daraus folgt die Notwendigkeit, ihnen Ressourcen zur Verfügung zu stellen, dieser Aufgabe gerecht zu werden. Dabei werden von FTI-politischen (Förderungs-)ExpertInnen zwei Argumentationslinien vertreten:

1. Fachhochschulen haben bereits den Zugang zu bestehenden Forschungsförderungsangeboten (COIN, Bridge, RSA etc.) und können sich mit anderen Zielgruppen im Wettbewerb ihr Forschungsknowhow aufbauen. Ein spezielles Angebot zur Förderung von Forschung an FH's in Kooperation mit Unternehmen müsse sich zumindest in einer bestehenden Programmlinie wiederfinden, damit hier nicht zusätzliche „Kleinprogramme“ aufgelegt werden.

2. Fachhochschulen benötigen ein eigenes, zielgruppenspezifisches Förderungsprogramm, das die Voraussetzungen für die längerfristige Durchführung von wissensintensiver, anwendungsorientierter Forschung schafft und hier einen substanziellen Mehrwert für Unternehmen und Region bietet. Bestehende Programme zielen vor allem auf die Forschungsleistung in Kooperation mit den Unternehmen, haben jedoch keine Rückwirkungen auf den institutionellen bzw. organisationalen Zusammenhang (wie z. B. Einbindung in die Lehre, Kooperation mit wissenschafltichen Einreichungen, Herstellen regionaler Verbundfähigkeit FH Unternehmen).

Bei den Josef Ressel Zentren ist es von Bedeutung, wie Forschung systematisch und strukturell an der FH verankert wird, wie der Forschungszentrumscharakter in der Region zum Tragen kommt und dieser weiterhin den Unternehmen zur Verfügung steht. Diese Aufgaben werden in keiner bestehenden Programmlinie von COIN abgebildet. Daher erscheint es nicht sinnvoll, eine - wie im Programmdokument geforderte - zukünftige Einbettung in COIN anzustreben. Es wird mit einer Einbindung in COIN kein Mehrwert für das Programm und seine Zielsetzungen erkannt, im Gegenteil, eher eine „Verwässerung“ der Zielsetzungen und Aufgabenbereiche sowie ein „down-grading“ in Richtung „Projekt“ erwartet.

\section{- Letztlich bleibt die Frage, ob das JRZ-Programm eine Förderungslücke schließt?}

Diese ist aufgrund der Erhebungen und Analysen im Rahmen der Programmevaluierung mit „.Ja“ zu beantworten. Es wird wissenschaftsintensive, anwendungsorientierte Forschung an FH's in Kooperation mit der Wirtschaft ermöglicht, die ein deutliches „up-grading“ bisheriger Forschungsleistungen der FH und Forschungsergebnisse für Unternehmen in der Region darstellt. Neben den direkten Effekten für die Forschung entstehen auch positive indirekte Effekte in Hinblick auf eine anreizinduzierte Profilbildung im FH Sektor und einer verbesserten Verbundfähigkeit FH Wirtschaft in der Region. 


\section{A.1. Anhang 1: Interviewte ExpertInnen}

\section{I Josef Ressel Zentren und Fachhochschulverantwortliche}

\begin{tabular}{l|l|l} 
Name & Funktion & JRZ/FH \\
\hline Dr. Michael Affenzeller & JRZ-Leiter & Heureka [FH OÖ] \\
\hline Dr. Johann Kastner & Forschungsleiter & FH OÖ \\
\hline Dr. Thomas Breuer. & JRZ Leiter & OptimUns [FH Vlbg] \\
\hline Dr. Hedwig Natterer & Geschäftsführung & FH Voralberg \\
\hline DI Christian Heschl & JRZ Leiter & CFD [FH Bgld.] \\
\hline $\begin{array}{l}\text { Mag. Ingrid Schwab- } \\
\text { Matkovits }\end{array}$ & Geschäftsführung & FH Burgenland
\end{tabular}

\section{I JRZ-beteiligte Unternehmen}

\begin{tabular}{l|l|l} 
Name & Unternehmen & JRZ/FH \\
\hline DI Christian Feilmeyer & Voestalpine Stahl GmbH & Heureka [FH OÖ] \\
\hline DI Gottfried Brunnbauer & Rosenbauer International & Heureka [FH OÖ] \\
\hline Markus Seeger & Hypo Landesbank Vlbg. & OptimUns [FH Vlbg] \\
\hline Alexander Kohler & Vorarlberger KraftwerkeAG & OptimUns [FHV] \\
\hline Martin Strele & Kairos & OptimUns [FHV] \\
\hline Dr. Günther Claus & Austrian Windpower & CFD [FH Bgld.] \\
\hline Helmut Jilg & Jilg GmbH & CFD [FH Bgld.] \\
\hline Dr. Günter Gronald & AE\&E Austria & CFD [FH Bgld.]
\end{tabular}

\section{I FTI-politische Akteure und Programmumsetzung}

\begin{tabular}{l|l|l} 
Name & Funktion & Organisation \\
\hline Dr. Knut Consemüller & Vorsitzender (Juli 2010) & RFTE \\
\hline Univ.Prof. Dr. Norbert Vana & Vizepräsident FHR & Fachhochschulrat \\
\hline Dr. Bruno Hribernik & Stv. Vorsitzender CDG & CDG \\
\hline Mag. Rudolf Lichtmannegger & Wirtschaftspolitik & WKO \\
\hline Dr. Roswitha Wiedenhofer & Forschungsmanagement & FH Joanneum \\
\hline Mag. Kurt Koleznik & Generalsekretär & FHK \\
\hline Dr. Rupert Pichler & Abtlg.Leiter Innovation & BMVIT \\
\hline Mag. Friedrich Faulhammer & Sektionschef & BMWF \\
\hline Mag. Michael Binder & Leiter Strategie & FFG \\
\hline DI Martin Reishofer & Strukturprogramme & FFG \\
\hline Mag. Barbara Klimon & Programmleitung JRZ & FFG
\end{tabular}




\section{A.2. Anhang 2: Dokumenten-, Literatur- und Quellenverzeichnis}

\section{Dokumente}

- Programmdokument JRZ, Leitfaden JRZ

- Fachgutachten zu den Einreichungen

- Leitfaden für die Jury, Ergebnis der Jurysitzung

- Leitfaden Berichtswesen JRZ

- Zwischenevaluierungsbericht der JRZ (soweit verfügbar)

- Erhebung über Dissertationsvorhaben und Publikationen der JRZ

- Diverse Dokumente der Zentren: Medienberichterstattungen, Präsentationsfilm etc.

- Programmbezogener Jahresbericht „Josef Ressel Zentren“ der FFG laut Rahmenvertrag 2008 und 2009

\section{Literatur- u. Quellenverzeichnis}

- Bundesgesetz über Fachhochschul-Studiengänge, BGBl. Nr. 340/1993, zuletzt geändert mit Bundesgesetz BGBl. I Nr. 89/2007

- BMUKK (2004): Fachhochschulentwicklungs- und -finanzierungsplan III 2005/6-2009/10

- Fachhochschulkonferenz (2009): FHK-Position zur FTI-Strategie des Bundes, 18.11.2009, veröffentlicht auf der website der FHK (www. fhk.ac.at)

- Studierendenzahlen der FH

- www.fhr.ac.at/fhr_inhalt/02_qualitaetssicherung/eckdaten_entwicklung.htm

- uni:data: http://eportal.bmbwk.gv.at/discoverer/viewer?\&cn=cf_a104\&nlsl=deat\&fm=p://eportal.bmbwk.gv.at/discoverer/viewer?\&_po=10000000001100111011000

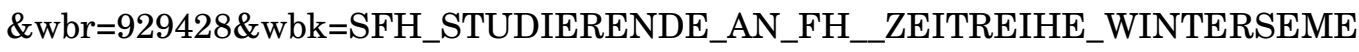
STER_PROD2 


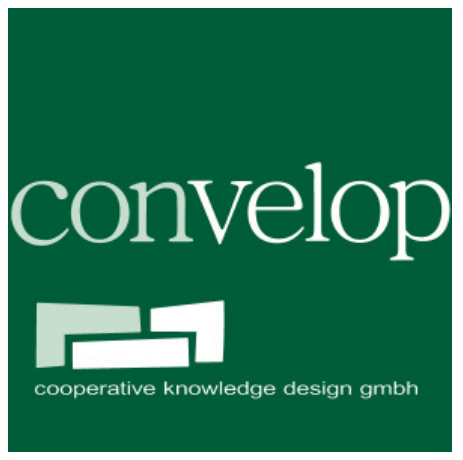

convelop

cooperative knowledge design gmbh

A-8010 Graz Bürgergasse 8-10/l Telefon: $\quad+43(0) 316720813$ Fax: $\quad+43(0) 316720813-20$ www.convelop.at office@convelop.at 